\title{
La place des céréales dans les habitudes alimentaires des peuples fenniques
}

\section{Suzanne Lesage}

\section{(2) OpenEdition}

1 Journals

Édition électronique

URL : https://journals.openedition.org/efo/5384

DOI : $10.4000 /$ efo. 5384

ISSN : 2275-1947

Éditeur

INALCO

Édition imprimée

ISBN : 978-2-343-08571-5

ISSN : 0071-2051

Référence électronique

Suzanne Lesage, "La place des céréales dans les habitudes alimentaires des peuples fenniques », Études finno-ougriennes [En ligne], 47 | 2015, mis en ligne le 01 juillet 2016, consulté le 20 septembre 2021. URL : http://journals.openedition.org/efo/5384 ; DOI : https://doi.org/10.4000/efo.5384

Ce document a été généré automatiquement le 20 septembre 2021.

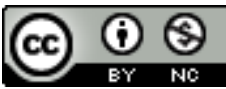

Études finno-ougriennes est mis à disposition selon les termes de la Licence Creative Commons Attribution - Pas d'Utilisation Commerciale 4.0 International. 


\title{
La place des céréales dans les habitudes alimentaires des peuples fenniques
}

\author{
Suzanne Lesage
}

1 Les céréales occupent une place très importante dans l'alimentation des peuples fenniques; en effet, il est relativement aisé de les conserver plusieurs mois (contrairement à la plupart des légumes), ce qui présente un avantage évident compte tenu de la rudesse des hivers. Que ce soit sous forme de farine ou bien de flocons, les céréales ne demandent qu'un taux d'humidité relativement faible, mais résistent bien aux grands froids comme aux grosses chaleurs (nettement plus rares dans la région).

2 De plus, le climat des régions en question ne permet pas la culture d'un grand nombre de légumes. Par ailleurs, les céréales permettent un apport énergétique conséquent : il faut garder à l'esprit qu'une partie de ces peuples est rurale et que la qualité nutritive des céréales leur permet d'obtenir les calories nécessaires aux rudes travaux physiques qu'impose l'activité agricole. Nous nous limiterons ici aux spécialités qui sont exclusivement ou presque composées de céréales, laissant de côté toutes les préparations mettant en jeu une quantité moindre, voire négligeable, de farine. Par ailleurs, les écrits scientifiques sur ce sujet sont très rares; nous nous appuierons donc en grande majorité sur des recueils de recettes ainsi que sur quelques ouvrages anthropologiques.

\section{Un aliment de base présentant un grand avantage : sa conservation}

On l'a dit, les céréales résistent bien aux grands froids comme aux grosses chaleurs. Par ailleurs, les Estoniens ont mis au point un mode de conservation spécifique étroitement lié à leur architecture traditionnelle. En effet, jusqu'au XVIII siècle, les gerbes de blé étaient accrochées en hauteur dans la pièce principale des fermes (rehetuba). Comme ce type d'habitation ne présentait pas de cheminée, la fumée du poêle était directement 
en contact avec le grain qui devenait ainsi «fumé ». Le fumage du blé lui permet de beaucoup mieux résister à l'humidité et réduit nettement le risque de fermentation.

Les différents peuples fenniques savent faire preuve d'une certaine originalité en ce qui concerne les modes de préparation de céréales.

\section{Les céréales consommées « telles quelles » : gruaux et flocons}

\subsection{Les gruaux}

5 Le gruau désigne les céréales dépouillées de leur enveloppe corticale par une mouture incomplète. Par extension, il désigne également la préparation de bouillie à partir de gruau. Les Estoniens consomment par exemple beaucoup de sarrasin (tatar) juste cuit à l'eau ou dans des soupes. Tout comme les Finlandais, ils en consomment encore aujourd'hui dès le petit-déjeuner sous forme de bouillies. Par ailleurs, la variété des céréales consommées en bouillie est très grande : orge, avoine, son, mais également, depuis le $\mathrm{XX}^{\mathrm{e}}$ siècle, on trouve très aisément des bouillies de semoule ou de riz.

\subsection{Les flocons d'avoine}

6 Les flocons d'avoine (kaer en estonien, herkkula en finnois) désignent l'avoine que l'on a vannée puis passée à la chaleur sèche; ensuite on lui retire la barbe avant de l'aplatir sous une presse.

7 On consomme ces flocons crus dans du yaourt, du lait ou du kefir, ou bien en bouillie.

\subsection{L'orge}

8 L'orge (oder en estonien, ohra en finnois) est l'une des premières céréales cultivées par l'homme. Elle est parfaitement adaptée au climat estonien (et à celui du sud de la Finlande) puisqu'elle résiste bien au froid. L'orge entière ne peut pas être consommée telle quelle, il faut la monder (retirer les enveloppes adhérentes, appelées glumes) ou la perler (retirer ces premières enveloppes ainsi que le son). Elle entre dans la composition de nombreux plats traditionnels estoniens: outre ceux que nous avons déjà mentionnés, l'orge est l'un des ingrédients essentiels de la soupe aux pois (hernesupp), soupe très nourrissante aux pois et au lard.

9 L'orge entre également dans la composition de deux plats traditionnels de la région de Viljandi : le mulgikapsas et le mulgipuder. Le premier plat réunit des choux acides (type choucroute) et des flocons d'orge; le tout est accompagné de viande de porc. La seconde recette associe la céréale à une purée de pommes de terre. Cette association féculent-céréale étonne souvent les palais français.

10 Comme nous l'avons vu précédemment, les céréales sont consommées quotidiennement, mais elles sont également la base de spécialités plus festives. Par exemple, on déguste en Estonie pour Noël du boudin (verivorst) dont la farce est principalement constituée d'orge. 


\section{Les farines} comme en Finlande, il est consommé en grande quantité à table et a presque le statut de plat à part entière. De plus, actuellement, en ville, il n'y a pas de réelle tradition du repas qui réunit la famille à heure fixe, même le soir. Il s'agit le plus souvent de légères collations prises seul ou à plusieurs; la tartine, plus ou moins élaborée, s'accorde donc très bien avec ce genre de repas. Dans les fermes, le soir, la famille se réunissait autour d'un repas, assez ritualisé (prières, silence), mais la tartine constituait la majorité des collations prises dans la journée quand les hommes passaient la journée dans les champs. Il est d'ailleurs en estonien, comme dans beaucoup d'autres langues, le symbole de la nourriture. On le retrouve dans de nombreuses expressions telles que jätku leiba (bon appétit, littéralement « que dure votre pain ») ou bien leiba luusse laskma (faire une pause digestive, littéralement « laisser le pain pénétrer jusqu'aux os »). 
l'on a prélevé lors de l'une des dernières étapes de la préparation du pain précédent. Les ingrédients de la recette de base sont simplement la farine de seigle, le ferment, du sucre et du sel. Toutefois, peu se contentent de cette recette : on ajoute très souvent de la farine maltée (farine faite à partir de grains germés) qui donne au pain un goût particulier et une couleur très foncée.

Jusqu'à la fin du premier millénaire de notre ère, le pain était fait exclusivement à base de farine d'orge, mais ce type de pain n'est plus consommé à présent. On préparait également des hädalaib (" pain des temps difficiles ») en ajoutant à la pâte de la mousse peignée, des baies ou de l'écorce d'arbre. Pendant les disettes, on préparait également des aganaleib, pain de seigle aigre dans la pâte duquel on ajoutait des aganad (balle de différentes céréales) réduites en purée. Jusqu'au XIX siècle, l'aganaleib était le pain quotidien et le pain de seigle (rukkileib) était réservé aux fêtes. C'est également à partir du XIX ${ }^{e}$ siècle que l'on voit apparaître sur les tables le pain blanc (sai). Le peenleib ou püülileib était fait à partir d'une farine très fine de seigle (rukkipüül) ou de farine simplement tamisée, que l'on mélangeait à de l'eau bouillante, mais sans juuretis. Le peenleib était le pain que l'on consommait aux fêtes dans le nord de l'Estonie au début, puis à la fin du XIX en Estonie du Sud, et plus tard encore sur les îles. Le keevaveeleib, pain sucré et doux à base de farine mélangé à de l'eau bouillante, à laquelle on ajoutait par la suite du juuretis, n'était consommé que sur les îles.

18 Au Sud de l'Estonie, on préparait également des kordleib qui étaient des pains garnis de chanvre, de lard, de viande ou de poisson.

On constate qu'à la fin du XIX ${ }^{e}$ siècle, le leib change de forme et de taille : les grandes miches rondes de deux ou trois kilos deviennent progressivement plus petites (500 g à peu près) et de forme allongée. Le leib était préparé dans un leivaküna (moule) fait à partir d'un seul et unique morceau de tronc taillé. On utilisait le bois d'un arbre feuillu (le plus souvent du bouleau ou du chêne) car celui-ci est rendait la préparation aigre. Aujourd'hui, ce type de moule n'est plus utilisé, on préfère les moules en terre cuite, dont l'entretien est beaucoup plus simple.

On l'agrémente également de graines (tournesol, lin, pépins de citrouille) ou bien d'épices. Ce pain est largement consommé en Estonie et dans l'Est de la Finlande. Il est assez proche du ХлЕБ russe tant sur le plan gustatif que linguistique (les deux mots ont été empruntés au mot allemand laib). Il est également très étroitement lié à l'architecture dans ces régions : en effet, le poêle qui permet de chauffer la maison fait également office de four et fonctionne quasiment en permanence. On y cuit le pain toutes les semaines (au maximum toutes les deux semaines, durée après laquelle le juuretis n'est plus actif).

21 À contrario, dans l'Ouest de la Finlande, le système de chauffage, tout comme en Suède, n'est pas associé au four. Le four est un petit bâtiment indépendant du lieu d'habitation et partagé le plus souvent par plusieurs familles. On utilise ce four deux ou trois fois par an. Le pain doit donc résister quatre à six mois. Sa composition n'est pas fondamentalement différente. En revanche, il est de forme ronde, très plat et percé au centre, et par nature très sec, ce qui permet une longue conservation. Le trou central permet de le faire passer dans une tige en bois que l'on accroche à l'horizontale en hauteur dans les maisons, afin que les souris ne puissent y avoir accès.

Dans le nord de la Finlande, les Sames, ne pouvant ni produire ni importer (ou en faible quantité) de farine de seigle ou de blé, consomment depuis le siècle dernier ce qu'on retrouve parfois sous la dénomination « pain nordique » ou « pain suédois » et que l'on 
appelle rieska en finnois. Cette spécialité est à base de farine d'orge, l'une des céréales qui résiste le mieux au froid.

En Finlande, probablement en raison de nombreux contacts avec les Suédois, on consomme également de petites « biscottes » au seigle très dures.

\subsection{Les préparations à base de farine de seigle}

On trouve également des préparations à base de croûte de seigle telle que les galettes caréliennes ou livoniennes (petites "pirogues" à base de farine de blé et de seigle fourrées dans le premier cas avec un porridge de riz et dans le second avec une purée de carottes et pommes de terre). On consomme souvent ces petites galettes accompagnées de beurre aux œufs (munavõi en estonien, munavõid en seto ou munavoi en finnois). Il s'agit de beurre (ou de margarine) mélangé à des œufs durs plus ou moins finement coupés. Les migrations caréliennes après la seconde guerre mondiale en Finlande ont permis aux particularités culinaires telles que ces galettes de se diffuser dans le pays.

Par ailleurs, comme la plupart des recettes évoquées ici, ces galettes se prêtent bien aux variations, on peut en imaginer de toute sorte concernant la garniture: purée de carottes, épinards, oignons, etc.

On peut également consommer en Finlande du kalakukko, spécialité que les Finlandais dégustent avec du porridge. La kalakukko est une croûte de seigle et d'avoine fourrée au poisson, remplie de morceaux de poissons comme la corégone, muikku en finnois (le plat s'appelle dans ce cas muikkukukko), le saumon (lohikukko), la perche (ahvenkukko). Les morceaux de poisson sont entourés de tranches de porc.

\subsection{Les gâteaux}

On prépare, en Estonie comme en Finlande, de nombreux gâteaux d'origine " occidentale ». Certains sont d'ailleurs considérés comme des plats nationaux. C'est notamment le cas du kringel estonien, brioche tressée et fourrée d'origine allemande. On peut également évoquer une spécialité plus fennique, le karask, préparation très simple, à mi-chemin entre le pain et le gâteau, à base de farine d'orge que l'on peut tartiner de beurre et de miel ou manger comme accompagnement de mets salés.

\section{La fermentation}

Comme nous l'avons vu, la fermentation est un élément central dans la fabrication du pain. On retrouve ce processus dans bon nombre de préparations.

\subsection{Le kile}

29 Certaines préparations requièrent que les céréales soient fermentées avant la préparation de la bouillie. Le kaarakiisla chez les Setos, kaerakile (ou kiisel) chez les Estoniens ou kiisel chez les Caréliens procèdent peu ou prou de la même recette : laisser reposer deux ou trois jours, dans de l'eau tiède, de la farine ou des flocons d'avoine (ou d'une autre céréale). Une fois que la préparation a fermenté, on la filtre et on recueille 
le liquide contenant le ferment (juuretis) que l'on fait bouillir comme pour la préparation d'une bouillie ordinaire. Ici, on fait une utilisation très originale de la céréale puisqu'elle n'est pas consommée à proprement parler. Il semble que ce type de préparation soit de moins en moins apprécié en Estonie. Ce mets, surtout en Estonie du Sud, était associé aux funérailles et aux commémorations. On en donnait souvent aux femmes qui venaient d'accoucher, mais il pouvait être consommé également sans raison particulière.

\subsection{Les boissons à base de céréales fermentées}

Le kali (équivalent du $K B A C$ « kvas » russe) est une boisson qui était très répandue dans l'Europe de l'Est et du Nord. Cette boisson est préparée à partir de la drêche, que l'on obtient en filtrant le moût lorsque l'on réalise de la bière, à laquelle on ajoute de l'eau et qu'on laisse fermenter et qui devient ainsi plus acide. À l'origine, cette boisson n'était produite que dans le Nord de l'Estonie, mais à partir de la fin du XIX ${ }^{\mathrm{e}}$ siècle, le kali s'est répandu dans le Sud du pays et sur les îles, où l'on appelait le breuvage taar. On peut également le préparer à base de jus de bouleau, de baies de genévrier ou bien de pain noir.

On a commencé à faire de la bière sur le territoire estonien il y a près de 2000 ans. Jusqu'au XIX ${ }^{e}$ siècle encore, on préparait la bière en versant de l'eau très chaude sur de la farine d'orge malté. Le mélange obtenu était appelé mesk («mêche» en français) et on le faisait cuire avec des pierres elles-mêmes brûlantes. Par la suite, la recette a été un peu modifiée : on verse maintenant de l'eau bouillante sur un mélange cuit de farine et d'eau (ôlleleib), et on ajoute à la préparation du houblon pour l'aromatiser. Aujourd'hui, en Estonie, les bières sont majoritairement à l'orge et au blé, mais on en trouve également au seigle.

Ainsi, comme nous avons pu le voir, les céréales occupent une place considérable dans l'alimentation des peuples fenniques. Ces derniers ont réussi à développer autour de cet ingrédient une gastronomie riche et variée, tant du point de vue du mode de transformation que du nombre de céréales utilisées. S'il apparaît que les céréales restent essentielles dans les habitudes alimentaires des Estoniens, des Finlandais et des Setos, il semble toutefois que les menus, particulièrement ceux des citadins, s'internationalisent peu à peu.

\section{BIBLIOGRAPHIE}

\section{Bibliographie}

CHASTANET Monique, SIGAUT François, 2010, Couscous, boulgour et polenta : transformer et consommer les céréales dans le monde, Paris : Karthala. 
GUERRIN Triinu, KARU Kadri, 2014, Võrokõisi köögi-ja söögiraamat (Le livre de cuisine et d'aliments des habitants du Võromaa), Võro Instituut.

JUHANSON Katariina, 1995, Soome-ugri kokaraamat (Livre de cuisine finno-ougrienne), Tallinn.

KALVIK Silvia, 1970, Eesti rahva toite (Les plats du peuple estonien), Tallinn : Valgus.

KARD Peter, 1994, Ungari köök, Maailma toite (Cuisine hongroise, plats du monde), Tallinn : Varrak.

LUIGAS Inara, 2002, Setu toidud (Plats seto), Põlva : Materra..

MOORA Aliise, 1991, Eesti Talurahva Vanem Toit I et II (Les anciens plats des paysans estoniens), Tallinn.

RÄNK Arvi, 1995, Etnograafia Sõnaraamat (Dictionnaire ethnographique), Tallinn.

TETRI Salme, 1994, Viiburi vanaema kokaraamat (Le livre de cuisine de la grand-mère de Viipuri), Tallinn : Sinisukk \& co.

TIIGI Liia, 1983, Leivatoite (Plats de pain), Tallinn : Valgus.

TROSKA Gea, VIIRES Ants, KARU Ellen, 2007, Eesti Rahvakultuuri Leksikon (Lexique de la culture populaire estonienne), Harjumaa.

VIIRES Ants, 2004, Vana Eesti Rahvaelu (La vie du peuple de l'ancienne Estonie), Tallinn.

\section{Sitographie}

http://www.suri.ee/koka/vepsaindex.html

Gastronomie des différents peuples finno-ougriens ; consulté en décembre 2014.

http://www.hkhk.edu.ee/vanker/eestitoit/

6_traditsioonilised_toidud_erinevates_eesti_piirkondades.html

Les différences régionales au sein de la gastronomie estonienne ; consulté en janvier 2015.

http://southestonia.ee/uploads/ckeditor/files/LET\%20toidutr\%C3\%BCkis\%202012\%20EST.pdf La gastronomie du sud de l'Estonie ; consulté en janvier 2016.

http://www.nami-nami.ee/

Recettes estoniennes ; consulté en janvier 2016.

http://www.france-estonie.org/article.php?id_article=82

Recette du karask ; consulté en mai 2015.

INDEX

Index géographique : Estonie, Finlande, Suède, Viljandi

motsclesru ПИТАНИЕ, КРУПЫ

motscleset toit, teraviljad

Keywords : Food, Crops

Mots-clés : alimentation, céréales

Index chronologique: XIXe siècle, XXe siècle, XXIe siècle

nomsmotscles Caréliens, Estoniens, Finlandais, peuples fenniques, Sames, Seto, Suédois 